\title{
Prevalência e Fatores Associados à Depressão e Ansiedade entre Estudantes Universitários da Área da Saúde de um Grande Centro Urbano do Nordeste do Brasil
}

Prevalence and Factors Associated with
Depression and Anxiety among University
Students in the Field of Health in a Large Urban
Center in the Northeast of Brazil

\section{PALAVRAS-CHAVE}

- Depressão.

- Ansiedade.

- Processo Saúde-Doença.

- Saúde Mental.

Andrea Mendes Leão

Ileana Pitombeira Gomes ${ }^{I I}$

Marcelo José Monteiro Ferreira ${ }^{I I}$

Luciano Pamplona de Góes CavalcantiII

\begin{abstract}
RESUMO
INTRODUÇÃO: Apesar dos altos índices de depressão e ansiedade, os profissionais de saúde ainda têm dificuldades em lidar com essas enfermidades, principalmente quando envolvem os estudantes universitários e profissionais da área da saúde. OBJETIVO: Estimar a prevalência e os fatores associados à depressão e ansiedade em estudantes universitários da área da saúde. MÉTODOS: Foi realizado um estudo transversal analítico com alunos do primeiro ano dos cursos da saúde (Biomedicina, Enfermagem, Fisioterapia, Medicina e Odontologia) de um Centro Universitário no Ceará. Foram aplicados três questionários. O primeiro envolvia aspectos sociodemográficos; o segundo foi o Inventário de Depressão Beck (BDI) (adaptação e padronização brasileira); e o terceiro, o Inventário de Ansiedade Beck (BAI) (adaptação e padronização brasileira). Os dados foram digitados utilizando-se o software Epi-info, versão 3.5.1. e analisados no Stata 11.2. Análise bivariada foi realizada para as associações entre as variáveis por meio da Razão de Prevalência (RP), sendo calculados seus respectivos intervalos de confiança e considerados significativos aqueles que apresentaram um $p<0,05$. O projeto foi aprovado pelo CEP, por meio do CAAE $n^{-}$1.132.140, de 30 de junho de 2015. RESULTADOS: Responderam aos questionários 476 estudantes. Predominou o sexo feminino $(71,6 \%)$, estudantes com menos de 20 anos de idade $(69,3 \%)$ e solteiros (92,0\%). As prevalências de depressão e ansiedade foram de 28,6\% e 36,1\%, respectivamente. Estudantes menos satisfeitos com o curso apresentaram chance quase quatro vezes maior de terem depressão $(p<0,001)$. Destacaram-se ainda fatores de risco como relacionamento familiar insatisfatório $(p<0,001)$, quantidade insuficiente de sono $(p=0,006) e$ relacionamento com amigos insatisfatório $(p<0,001)$. A prevalência de ansiedade esteve mais associada ao sexo feminino $(p<0,001)$ e entre os estudantes que apresentaram relacionamento insatisfatório com familiares $(p<0,001)$, amigos $(p=0,005)$ e colegas $(p<0,001)$. Apresentar insônia $(p<0,001)$, não fazer atividade física $(p=0,040)$ e maior preocupação com o futuro $(p=0,002)$ também apresentaram associação significativa com um quadro de ansiedade. CONCLUSÃO: As prevalências de ansiedade e depressão entre os estudantes da área da saúde foram muito superiores às da população em geral, tendo os estudantes do curso de Fisioterapia apresentado o resultado mais alto.
\end{abstract}




\section{KEY-WORDS}

- Depression.

- Anxiety.

- Health-Disease Process.

- Mental Health.

Recebido em: 19/6/18

Aceito em: 9/7/18
INTRODUCTION: Despite the high levels of depression and anxiety, health professionals still have difficulties when dealing with these illnesses, particularly when they involve university students and professionals in the field of health. OBJECTIVE: To estimate the prevalence and the factors associated with depression and anxiety among university students in the field of health. METHODS: A transversal, analytical study involving students in the first year of a degree in health (biomedicine, nursing, physiotherapy, medicine and odontology) at a University Center in Ceará. Three questionnaires were applied. The first involved sociodemographic aspects, the second was the Beck Depression Inventory - BDI, and the third was the Beck Anxiety Inventory - BAI (both adapted and standardized for Brazil). The data were digitalized using the software Epi-info version 3.5.1, and analyzed in Stata 11.2. Bivariate analysis was performed for the associations between the variables by means of the Prevalence Ratio (PR), as well as their respective intervals of confidence, with a level of significance of $p<0.05$. The project was approved by the REC, with CAAE number 1.132.140, of June 30, 2015. RESULTS: 476 students answered the questionnaires. The female sex was prevalent $(71.6 \%)$, with the majority of students under 20 years of age $(69.3 \%)$, and single $(92.0 \%)$. The prevalence rates for depression and anxiety were $28.6 \%$ and $36.1 \%$, respectively. Students who were less satisfied with the course had a four times higher likelihood of having depression $(p<0.001)$. Risk factors were highlighted, such as unsatisfactory family relationships $(p<0.001)$, lack of sleep $(p=0.006)$, and unsatisfactory relationships with friends $(p<0.001)$. The prevalence of anxiety was more closely associated with the female sex $(p<0.001)$, and with unsatisfactory relationships with family members $(p<0.001)$, friends $(p=0.005)$ and colleagues $(p<0.001)$. Having insomnia $(p<$ 0.001), not doing much physical activity $(p=0.040)$, and a higher concern about the future ( $p=$ 0.002) also presented significant association with anxiety status. CONCLUSION: The significant prevalence of anxiety and depression among students in the field of health were much higher than among the general population, with physiotherapy students having the highest result.

\section{INTRODUÇÃO}

Aproximadamente 450 milhões de pessoas sofrem de perturbações mentais ou neurobiológicas no mundo ${ }^{1}$. Destaca-se a depressão, considerada a principal causa de incapacitação no mundo e com possibilidade de se tornar a segunda maior carga de doença até $2030^{1,2}$. Em segundo lugar apresenta-se a ansiedade, comumente associada aos casos de depressão e que tem um efeito importante na diminuição da qualidade de vida das pessoas ${ }^{3-5}$.

A depressão é considerada um transtorno multifatorial, apresentando fatores de risco conhecidos, como afetividade negativa, experiências adversas na infância, eventos estressantes, familiares de primeiro grau com diagnóstico, transtornos subjacentes, condições médicas crônicas ou incapacitantes e que acomete cerca de $5,8 \%$ da população brasileira ${ }^{1,6-8}$. Caracteriza-se por tristeza ou irritabilidade, desinteresse ou desprazer, sentimento de culpa ou baixa autoestima, distúrbios do sono ou apetite, fadiga, dificuldades cognitivas e ideias recorrentes de morte ${ }^{9,10}$.
A ansiedade se caracteriza como uma emoção própria da vivência humana, sendo considerada uma reação natural e fundamental à autopreservação, mesmo gerando sensações de apreensão e alterações físicas desagradáveis ${ }^{7,11}$. Por outro lado, em sua condição patológica, apresenta-se de forma mais frequente e intensa, com sintomas que podem causar grande sofrimento e prejuízo na vida cotidiana, como evasão escolar, abandono de emprego e abuso de substâncias ${ }^{8,12,13}$.

Entre os vários tipos de transtornos destaca-se o de Ansiedade Social (TAS) e o de Ansiedade Generalizada (TAG), que trazem dificuldades ao convívio social e aos aspectos da vida em geral, além daqueles inerentes ao desempenho dos acadêmicos ${ }^{1,9,14,15}$. Nos últimos anos, a saúde mental desses estudantes universitários tornou-se foco de atenção não só dos especialistas da área de saúde, mas da sociedade em ge$\mathrm{ral}^{16}$, pois o sofrimento emocional do estudante da saúde não se limita a ele próprio, mas tem impacto emocional sobre sua relação com os pacientes ${ }^{17,18}$. Esses estudantes universitários 
da área da saúde geralmente não recebem formação adequada sobre os tópicos de saúde mental e frequentemente se expõem a situações estressantes, o que pode levá-los a um mau desempenho acadêmico, adoecimento psíquico, risco de suicídio ou dificuldade no tratamento de doentes ${ }^{17,19-21}$.

Dessa forma, o objetivo deste trabalho foi estimar a prevalência e os fatores associados à depressão e ansiedade entre estudantes universitários da área da saúde de um Centro Universitário no Nordeste do Brasil.

\section{MÉTODOS}

Tipo de estudo

Trata-se de um estudo transversal analítico, realizado numa instituição de ensino superior do Nordeste do Brasil.

\section{População do estudo}

Foram investigados estudantes maiores de 18 anos, regularmente matriculados nos cursos da área da saúde (Biomedicina, Enfermagem, Fisioterapia, Medicina e Odontologia) e que frequentavam o primeiro ano do curso (primeiro e segundo semestres).

\section{Coleta de dados}

Foi aplicado um questionário elaborado pelos pesquisadores, além do Inventário de Depressão Beck (BDI), na sua adaptação e padronização brasileira, e o Inventário de Ansiedade Beck (BAI), também na sua adaptação e padronização brasileira.

No questionário constavam questões relativas a características sociodemográficas (sexo, idade, local de procedência, com quem reside e quantas pessoas residem na casa); sociais (emprego, renda familiar, plano de saúde e religião); e comportamentais (quantidade de sono, atividade física, tempo de lazer, uso de cigarro, ingestão de álcool e nível de satisfação com o curso).

O Inventário de Depressão Beck (BDI), criado originalmente para avaliar o nível de depressão em pacientes psiquiátricos ${ }^{22}$, passou a ser utilizado tanto na área clínica como na pesquisa, mostrando-se um instrumento útil também para a população em geral, apresentando alta confiabilidade e validade quando comparado a outros instrumentos ${ }^{22,23}$. Ele avalia aspectos como: tristeza, pessimismo, sentimento de fracasso, insatisfação, culpa, punição, autoaversão, autoacusações, ideias suicidas, choro, irritabilidade, retraimento social, indecisão, mudança na autoimagem, dificuldade de trabalhar, insônia, fatigabilidade, perda de apetite, perda de peso, preocupações somáticas e perda de libido ${ }^{22}$. É composto por uma escala de 21 itens, com quatro opções para cada item, em graus crescentes de gravidade, com escores de 0 a 3.
O Inventário de Ansiedade Beck (BAI) foi criado com o objetivo inicial de medir a intensidade dos sintomas de ansiedade $^{24}$, sendo amplamente utilizado e validado tanto para pacientes psiquiátricos, como para a população em geral ${ }^{22}$. É constituído por 21 itens que devem ser avaliados numa escala de quatro pontos, refletindo níveis de gravidade crescentes - absolutamente não, levemente, moderadamente, gravemente -, sendo que o escore total é a soma dos escores dos itens individuais.

Ambos os instrumentos de coleta foram aplicados durante o final do semestre 2015.2 e começo do 2016.1.

\section{Controle de qualidade}

$\mathrm{O}$ instrumento de coleta de dados foi submetido a um estudo piloto para testar a compreensão sobre as questões e a aplicabilidade ao público específico. Os profissionais que aplicaram o questionário foram treinados, e antes da digitação dos dados estes foram revisados. Foram excluídos os questionários rasurados e que comprometessem o somatório dos escores dos instrumentos utilizados. Para a entrada dos dados e checagem da consistência, foi utilizado o software Epi-info.

\section{Análise dos dados}

Os dados foram digitados utilizando-se o software Epi-info, versão $3.5 .1^{\circledR}$, e analisados no Stata $11.2^{\circledR}$.

Para o BDI, foram somados os escores individuais e posteriormente adotados os seguintes pontos de corte: mínimo, variando de zero a 11; leve, entre 12 e 19; moderado, variando entre 20 e 35; e grave, entre 36 e 63 . O resultado mínimo foi considerado como ausência de depressão ${ }^{22}$. Para os dados do BAI, foram adotados os pontos de corte: mínimo, variando de zero a 10; leve, entre 11 e 19; moderado, variando entre 20 e 30; e grave, entre 31 e 63 . O resultado mínimo foi considerado como ausência de ansiedade ${ }^{22}$.

Foram utilizados testes paramétricos (teste $\mathrm{t}$ de Student) e não paramétricos (qui-quadrado de Pearson) de acordo com as características das variáveis do estudo. Análise bivariada foi realizada para as associações entre as variáveis, sendo calculadas, também, as razões de prevalência, bem como seus respectivos intervalos de confiança. Para isso, foi considerado um intervalo de confiança de $95 \%$, e resultados significativos aqueles que apresentaram um $\mathrm{p}<0,05$.

\section{Aspectos éticos}

O estudo seguiu os princípios éticos de acordo com a Resolução 466/12 do Conselho Nacional de Saúde, tendo sido aprovado pelo Comitê de Ética e Pesquisa com o parecer 1.132.140, de 30 de junho de 2015. O TCLE foi assinado por todos os estudantes após apresentação dos objetivos da pesquisa, respei- 
tando e assegurando a adequação às peculiaridades culturais e linguísticas dos envolvidos.

\section{RESULTADOS}

\section{Caracterização da amostra}

Considerando os critérios de inclusão, 649 estudantes eram elegíveis para participar da pesquisa. Foram respondidos 579 questionários $(89,2 \%)$, e 476 (82,7\%) foram considerados válidos. Os cursos com maior número proporcional de questionários respondidos foram Fisioterapia (103/104; 99,0\%); Odontologia (214/226; 94,6\%); Medicina (159/179; 88,8\%), Biomedicina ( $59 / 78 ; 75,6 \%$ ) e Enfermagem com $44 / 62 ; 70,9 \%$ ).

Predominou o sexo feminino com $71,6 \% ; 69,3 \%$ dos estudantes tinham menos de 20 anos de idade, e 92,0\% eram solteiros. A maior parte não exercia atividade remunerada $(80,7 \%)$; $57,1 \%$ tinham renda familiar média de 7,5 salários mínimos; $77,9 \%$ residiam com os pais ou cônjuges, e 88,2\% declararam ter alguma religião. Ingestão de álcool foi citada por 53,4\% dos alunos. A maioria relatou não fumar (96,8\%); 87,6\% se declararam insatisfeitos com a quantidade de sono; $87,8 \%$ faziam atividade física, e 74,1\% consideraram que tinham boa saúde. A maior parte dos alunos declarou estar satisfeita com o curso escolhido (98,9\%), mas 73,9\% manifestaram preocupação com o futuro profissional (Tabela 1).

\section{Prevalência de depressão e ansiedade}

A prevalência de depressão entre os estudantes foi de 28,6\%, variando entre o curso de Fisioterapia, com 35,7\%, e o curso de Enfermagem, com 15,0\%. Entre os estudantes com algum nível de depressão destacou-se que 103 (75,8\%) tinham nível leve, 32 (23,5\%) nível moderado e $1(0,7 \%)$ apresentou a forma grave (Tabela 2).

Já a prevalência de ansiedade foi de 36,1\%, variando entre $52,4 \%$ para o curso de Fisioterapia e $25,9 \%$ para o curso de

\begin{tabular}{|c|c|c|c|c|c|c|}
\hline & $\begin{array}{l}\text { os socioecon } \\
\text { área da saúd }\end{array}$ & $\begin{array}{l}\text { os e condiçã } \\
\text { uma institui }\end{array}$ & $\begin{array}{l}\text { BELA } 1 \\
\text { ria de saúde } \\
\text { e ensino sup }\end{array}$ & $\begin{array}{l}\text { studantes } \\
\text { do Nordes }\end{array}$ & $\begin{array}{l}\text { meiro ano da } \\
\text { Brasil }\end{array}$ & \\
\hline $\begin{array}{l}\text { Variáveis } \\
\text { socioeconômicas }\end{array}$ & $\begin{array}{c}\text { Biomedicina } \\
\text { N (\%) }\end{array}$ & $\begin{array}{c}\text { Enfermagem } \\
\text { N }(\%)\end{array}$ & $\begin{array}{c}\text { Fisioterapia } \\
\text { N (\%) }\end{array}$ & $\begin{array}{c}\text { Medicina } \\
\text { N (\%) }\end{array}$ & $\begin{array}{l}\text { Odontologia } \\
\text { N (\%) }\end{array}$ & $\begin{array}{l}\text { Total } \\
\text { N (\%) }\end{array}$ \\
\hline Sexo & & & & & & \\
\hline Feminino & $31(64,6)$ & $38(95,0)$ & $67(79,8)$ & $82(55,8)$ & $123(78,3)$ & $341(71,6)$ \\
\hline Masculino & $17(35,4)$ & $2(5,0)$ & $17(20,2)$ & $65(44,2)$ & $34(21,7)$ & $135(28,4)$ \\
\hline $\begin{array}{l}\text { Mediana idade } \\
\text { (mín.-máx.) }\end{array}$ & $\begin{array}{c}19,0 \\
(18-41)\end{array}$ & $\begin{array}{c}21,5 \\
(18-59)\end{array}$ & $\begin{array}{c}19,0 \\
(18-42)\end{array}$ & $\begin{array}{c}19,0 \\
(18-35)\end{array}$ & $\begin{array}{c}19,0 \\
(18-38)\end{array}$ & $\begin{array}{c}19,0 \\
(18-59)\end{array}$ \\
\hline Estado civil & & & & & & \\
\hline Outros & $4(8,3)$ & $11(27,5)$ & $8(9,5)$ & $3(2,0)$ & $12(7,6)$ & $38(8,0)$ \\
\hline Solteiro & $44(91,7)$ & $29(72,5)$ & $76(90,5)$ & $144(98,0)$ & $145(92,4)$ & $438(92,0)$ \\
\hline Tem alguma relig & & & & & & \\
\hline Não & $10(20,8)$ & $4(10,0)$ & $9(10,7)$ & $11(7,5)$ & $22(14,0)$ & $56(11,8)$ \\
\hline Sim & $38(79,2)$ & $36(90,0)$ & $75(89,3)$ & $136(92,5)$ & $135(86,0)$ & $420(88,2)$ \\
\hline Renda familiar $\mathrm{m}$ & & & & & & \\
\hline Salário mínimo & 5,5806 & 3,7813 & 4,1385 & 13,0698 & 6,5250 & 7,5136 \\
\hline Mora com & & & & & & \\
\hline Outros & $6(12,5)$ & $5(12,8)$ & $18(21,7)$ & $29(19,9)$ & $46(29,7)$ & $104(22,1)$ \\
\hline Pais/cônjuge & $42(87,5)$ & $34(87,2)$ & $65(78,3)$ & $117(80,1)$ & $109(70,3)$ & $367(77,9)$ \\
\hline Realiza alguma at & emunerada & & & & & \\
\hline Não & $37(77,1)$ & $17(43,6)$ & $59(72,0)$ & $138(97,2)$ & $122(80,8)$ & $373(80,7)$ \\
\hline Sim & $11(22,9)$ & $22(56,4)$ & $23(28,0)$ & $4(2,8)$ & $29(19,2)$ & $89(19,3)$ \\
\hline Tem plano de saú & & & & & & \\
\hline Não & $15(31,2)$ & $16(40,0)$ & $32(38,1)$ & $12(8,2)$ & $52(33,1)$ & $127(26,7)$ \\
\hline Sim & $33(68,8)$ & $24(60,0)$ & $52(61,9)$ & $135(91,8)$ & $105(66,9)$ & $349(73,3)$ \\
\hline Fuma & & & & & & \\
\hline Não & $42(87,5)$ & $39(97,5)$ & $82(97,6)$ & $144(98,0)$ & $154(98,1)$ & $461(96,8)$ \\
\hline Sim & $6(12,5)$ & $1(2,5)$ & $2(2,4)$ & $3(2,0)$ & $3(1,9)$ & $15(3,2)$ \\
\hline Bebe & & & & & & \\
\hline Não & $18(37,5)$ & $27(67,5)$ & $41(48,8)$ & $61(41,5)$ & $75(47,8)$ & $222(46,6)$ \\
\hline Sim & $30(62,5)$ & $13(32,5)$ & $43(51,2)$ & $86(58,5)$ & $82(52,2)$ & $254(53,4)$ \\
\hline Pratica alguma at & sica & & & & & \\
\hline Não & $2(4,2)$ & $6(15,0)$ & $12(14,3)$ & $16(10,9)$ & $22(14,0)$ & $58(12,2)$ \\
\hline Sim & $46(95,8)$ & $34(85,0)$ & $72(85,7)$ & $131(89,1)$ & $135(86,0)$ & $418(87,8)$ \\
\hline
\end{tabular}

$\left.58\right|_{42(4): 55-65 ; 218} ^{\text {REVISTA BRASILEIRA DE EDUCAÇÃO MÉDICA }}$ 
Medicina. Entre os alunos considerados com algum nível de ansiedade, 62,2\% apresentaram a forma leve, 27,9\% nível moderado e 9,9\% nível considerado grave (Tabela 2).

Considerando os diferentes cursos e o nível de depressão, as formas leves foram mais prevalentes no curso de Enfermagem $(83,3 \%)$, moderada no curso de Fisioterapia (30\%) e grave entre os estudantes de Biomedicina (5,9\%). Os alunos mais ansiosos estavam nos cursos de Fisioterapia (52,4\%), seguidos dos alunos de Odontologia (38,9\%) e Biomedicina (33,3\%). O curso de Medicina apareceu em quinto lugar, com um percentual de 25,9\%. Já em relação ao nível de ansiedade, obteve-se maior prevalência do leve na Enfermagem (76,9\%), moderado na Biomedicina (31,3\%) e sua forma grave no curso de Fisioterapia $(15,9 \%)$ (Tabela 2$)$.

Fatores associados ao risco de depressão

Vários fatores foram associados a maior prevalência de depressão entre os estudantes. Destacam-se os alunos menos sa- tisfeitos com o curso, que apresentaram chance quase quatro vezes maior de terem depressão quando comparados aos que estavam satisfeitos com o curso escolhido ( $R P=3,59$; IC: 3,10$4,15 ; \mathrm{p}<0,001)$. Destacaram-se ainda com chances pelo menos duas vezes maior de ocasionar depressão variáveis como relacionamento familiar ( $\mathrm{RP}=2,70$; IC: 2,06-3,53; $\mathrm{p}<0,001$ ), quantidade insuficiente de sono $(\mathrm{RP}=2,27$; IC: $1,17-4,40 ; \mathrm{p}=$ $0,006)$ e relacionamento com amigos ( $R P=2,16$; IC: 1,49-3,13; $\mathrm{p}<0,001$ ) (Tabela 3). Foram associadas estatisticamente à presença de depressão as variáveis relacionamento insatisfatório com colegas do curso ( $\mathrm{p}<0,001)$ e com docentes ( $\mathrm{p}=0,004)$, além de não estar no curso escolhido como primeira opção ( $p$ $=0,019)$ e não praticar atividade física $(\mathrm{p}=0,003)$. As variáveis sexo, faixa etária, estado civil, religião, renda, com quem reside, ter plano de saúde, motivo da escolha do curso, forma de pagamento do curso, se fuma, bebe ou se há preocupação com o futuro não apresentaram associações significativas com a presença de depressão (Tabela 3).

\begin{tabular}{|c|c|c|c|c|c|c|}
\hline \multicolumn{7}{|c|}{$\begin{array}{c}\text { TABELA } 2 \\
\text { Fatores ligados ao curso, prevalência de depressão e ansiedade dos estudantes de primeiro } \\
\text { ano da área da saúde de uma instituição de ensino superior do Nordeste do Brasil }\end{array}$} \\
\hline Variáveis & $\begin{array}{c}\text { Biomedicina } \\
\text { N (\%) }\end{array}$ & $\begin{array}{c}\text { Enfermagem } \\
\text { N (\%) }\end{array}$ & $\begin{array}{c}\text { Fisioterapia } \\
\text { N (\%) }\end{array}$ & $\begin{array}{l}\text { Medicina } \\
\text { N (\%) }\end{array}$ & $\begin{array}{c}\text { Odontologia } \\
\text { N (\%) }\end{array}$ & $\begin{array}{l}\text { Total } \\
\text { N (\%) }\end{array}$ \\
\hline \multicolumn{7}{|c|}{ Forma de ingresso no curso } \\
\hline Outros & $3(6,2)$ & $6(15,0)$ & $5(6,0)$ & $6(4,1)$ & $3(1,9)$ & $23(4,8)$ \\
\hline Vestibular & $45(93,8)$ & $34(85,0)$ & $79(94,0)$ & $141(95,9)$ & $154(98,1)$ & $453(95,2)$ \\
\hline \multicolumn{7}{|c|}{ Curso como primeira opção } \\
\hline Não & $33(68,8)$ & $6(15,4)$ & $39(46,4)$ & $15(10,2)$ & $59(37,8)$ & $152(32,1)$ \\
\hline Sim & $15(31,2)$ & $33(84,6)$ & $45(53,6)$ & $132(89,8)$ & $97(62,2)$ & $322(67,9)$ \\
\hline \multicolumn{7}{|c|}{ Motivo da escolha do curso } \\
\hline Outros & $18(39,1)$ & $4(14,3)$ & $28(34,1)$ & $27(21,3)$ & $49(32,5)$ & $126(29,0)$ \\
\hline Vocação & $28(60,9)$ & $24(85,7)$ & $54(65,9)$ & $100(78,7)$ & $102(67,5)$ & $308(71,0)$ \\
\hline \multicolumn{7}{|c|}{ Satisfação com o curso } \\
\hline Não & $1(2,1)$ & $0(0,0)$ & $0(0,0)$ & $1(0,7)$ & $3(1,9)$ & $5(1,1)$ \\
\hline Sim & $47(97,9)$ & $40(100,0)$ & $84(100,0)$ & $146(99,3)$ & $154(98,1)$ & $471(98,9)$ \\
\hline \multicolumn{7}{|c|}{ Preocupação com o futuro profissional } \\
\hline Não & $13(27,1)$ & $6(15,0)$ & $17(20,2)$ & $55(37,4)$ & $33(21,0)$ & $124(26,1)$ \\
\hline Sim & $35(72,9)$ & $34(85,0)$ & $67(79,8)$ & $92(62,6)$ & $124(79,0)$ & $352(73,9)$ \\
\hline \multicolumn{7}{|c|}{ Tem depressão } \\
\hline Não & $31(64,6)$ & $34(85,0)$ & $54(64,3)$ & $109(74,1)$ & $112(71,3)$ & $340(71,4)$ \\
\hline Sim & $17(35,4)$ & $6(15,0)$ & $30(35,7)$ & $38(25,9)$ & $45(28,7)$ & $136(28,6)$ \\
\hline \multicolumn{7}{|c|}{ Nível de depressão } \\
\hline Leve & $13(76,5)$ & $5(83,3)$ & $21(70,0)$ & $30(78,9)$ & $34(75,6)$ & $103(75,8)$ \\
\hline Moderado & $3(17,6)$ & $1(16,7)$ & $9(30,0)$ & $8(21,1)$ & $11(24,4)$ & $32(23,5)$ \\
\hline Grave & $1(5,9)$ & $0(0,0)$ & $0(0,0)$ & $0(0,0)$ & $0(0,0)$ & $1(0,7)$ \\
\hline \multicolumn{7}{|c|}{ Tem ansiedade } \\
\hline Não & $32(66,7)$ & $27(67,5)$ & $40(47,6)$ & $109(74,1)$ & $96(61,1)$ & $304(63,9)$ \\
\hline Sim & $16(33,3)$ & $13(32,5)$ & $44(52,4)$ & $38(25,9)$ & $61(38,9)$ & $172(36,1)$ \\
\hline \multicolumn{7}{|c|}{ Nível de ansiedade } \\
\hline Leve & $9(56,2)$ & $10(76,9)$ & $25(56,8)$ & $27(71,0)$ & $36(59,0)$ & $107(62,2)$ \\
\hline Moderado & $5(31,3)$ & $2(15,4)$ & $12(27,3)$ & $10(26,3)$ & $19(31,1)$ & $48(27,9)$ \\
\hline Grave & $2(12,5)$ & $1(7,7)$ & $7(15,9)$ & $1(2,7)$ & $6(9,9)$ & $17(9,9)$ \\
\hline
\end{tabular}

$59 \mid \begin{array}{ll}\text { REVISTA BRASILEIRA DE EDUCAÇÃO MÉDICA } \\ 42(4): 55-65 ; 2018\end{array}$ 


\section{TABELA 3}

Fatores associados à depressão dos estudantes de primeiro ano da área da saúde de uma instituição de ensino superior do Nordeste do Brasil

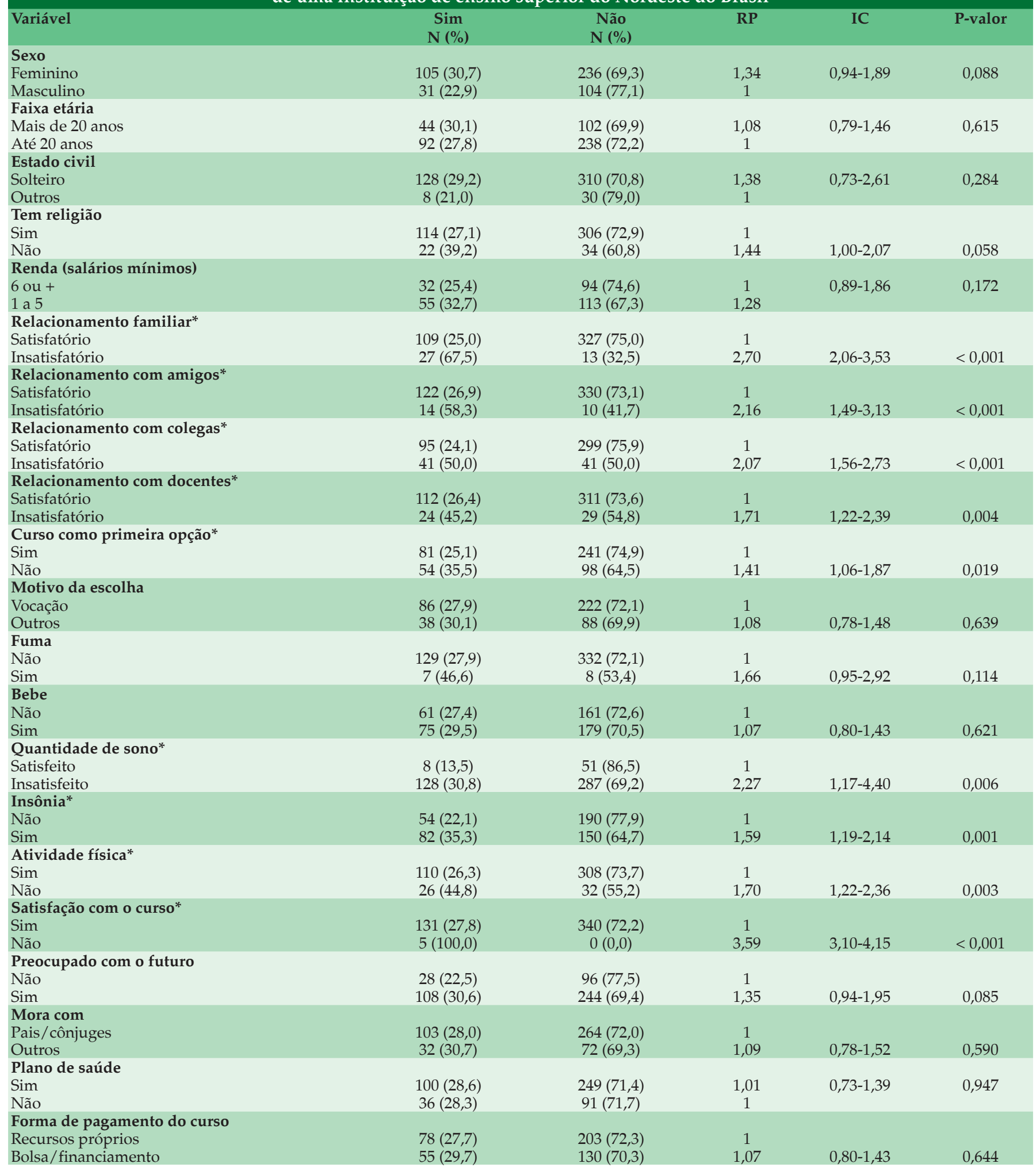

* Variáveis com significância estatística $(\mathrm{p}<0,05)$. 
Fatores associados ao risco de ansiedade

A prevalência de ansiedade esteve mais associada ao sexo feminino $(R P=1,66 ; p<0,001)$. Foi significativamente mais presente entre os estudantes que apresentaram relacionamento insatisfatório com familiares ( $p<0,001)$, amigos $(p=0,005)$ e colegas ( $p<0,001)$. Variáveis como ter insônia ( $p<0,001)$, não fazer atividade física $(p=0,040)$, maior preocupação com o futuro ( $p=0,002)$ também apresentaram associação significativa com um quadro de ansiedade (Tabela 4).

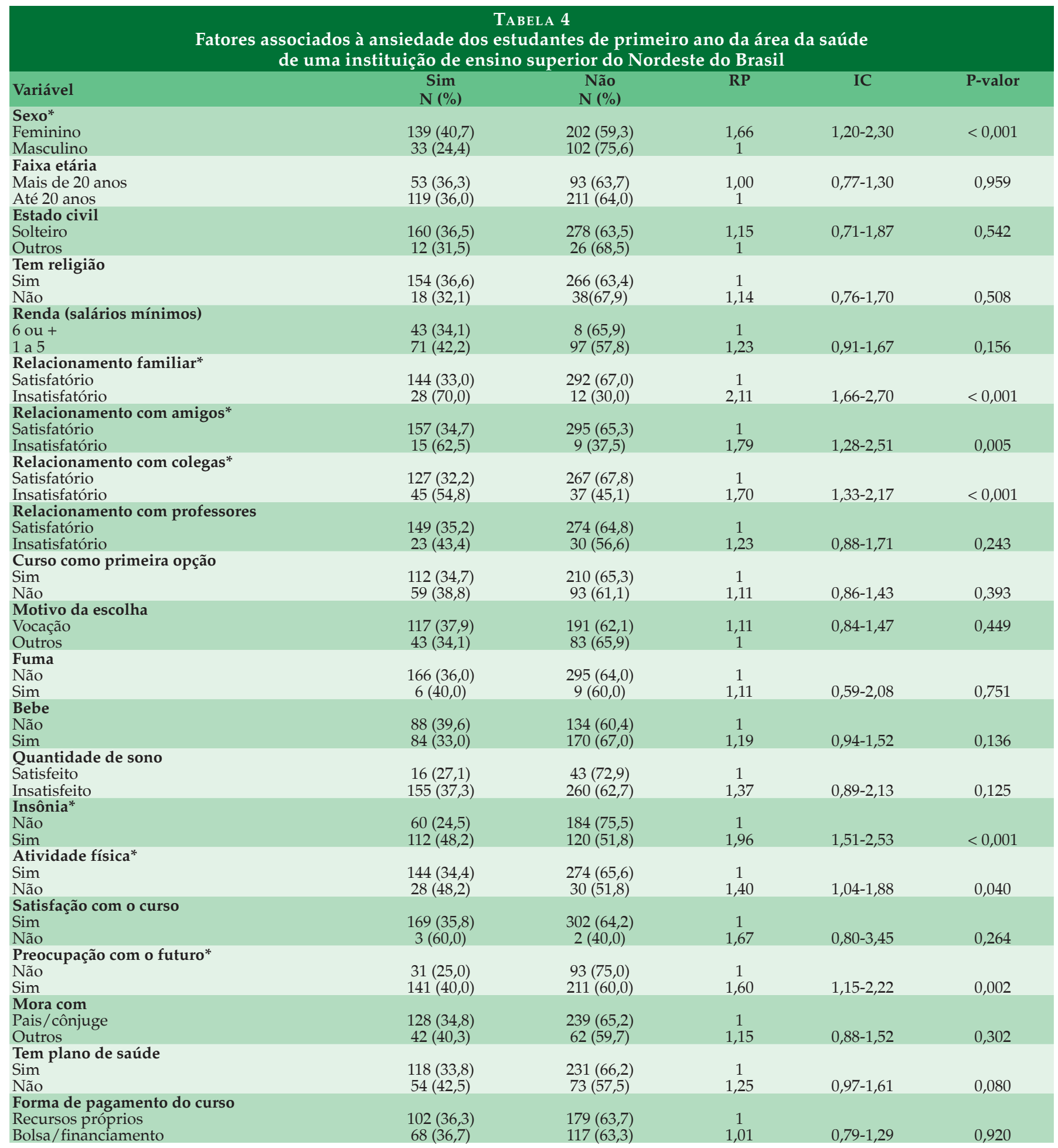




\section{DISCUSSÃO}

A prevalência de depressão e ansiedade entre os estudantes da área da saúde foi muito superior à de estudantes de outras áreas e da população em geral no Brasil ${ }^{15,25,26}$, com destaque para o curso de Fisioterapia. Chama a atenção o fato de o curso de Medicina, embora seja o mais investigado no tocante a esses aspectos, ter ocupado o quarto lugar em níveis de depressão e o quinto em ansiedade quando comparado aos outros cursos da área da saúde. Entretanto, apresenta resultados bem acima daqueles da população em geral, o que merece ser considerado um importante problema para a comunidade médica e acadêmica.

A prevalência de depressão em todos os cursos foi mais elevada no sexo feminino. Em Goiás, Uberlândia e Ribeirão Preto, essa associação também ocorreu ${ }^{16,27,28}$. Entretanto, em trabalho realizado em Minas Gerais com estudantes de Odontologia, não ocorreu o mesmo ${ }^{29}$. O resultado desse último estudo difere do aqui apresentado e do encontrado na literatura nacional e internacional, que aponta maior prevalência de depressão no sexo feminino na população geral ${ }^{30,31}$, independentemente de idade e se estudante da área da saúde ou não.

Maior prevalência de depressão esteve mais associada aos estudantes que citaram ter relacionamentos insatisfatórios com familiares, amigos, colegas de sala e professores $(\mathrm{p}<0,05)$. Estudos em Santa Catarina, Amapá, Ceará e Rio de Janeiro trazem análises semelhantes, corroborando a afirmação de que laços afetivos saudáveis são fundamentais à saúde emocional dos estudantes ${ }^{32-35}$. Esses estudos complementam as inúmeras teorias psicológicas que estudam a importância das relações afetivas para a saúde emocional ${ }^{36,37}$.

Insatisfação com quantidade de sono, insônia e inatividade física foram aspectos fortemente associados à prevalência de depressão entre os estudantes. Em uma Universidade de Ribeirão Preto, foi encontrado distúrbio do sono entre aqueles que apresentavam depressão moderada ${ }^{28}$, e no Ceará já foi documentada associação significativa entre depressão e insônia em universitários do primeiro ano ${ }^{32}$. O presente estudo corrobora a literatura médica disponível, que aponta a importância do sono para a saúde física e emocional do indivíduo, já que noites maldormidas e inatividade física podem gerar problemas como fadiga, irritabilidade, hipertensão arterial, dificuldades de atenção e de memória, e transtornos mentais como depressão e ansiedade $\mathrm{e}^{26,38,39}$.

O estudo aqui apresentado corrobora essa análise e soma-se à vasta literatura que incentiva a importância da prática de atividade física para a manutenção da saúde e controle do estresse, bem como indica essa prática como coadjuvante no tratamento da depressão ${ }^{40,41}$. Quando praticada com regula- ridade, auxilia o organismo na produção de endorfina e serotonina, neurotransmissores responsáveis pela sensação de prazer e bem-estar. Também de forma indireta, por meio das transformações corporais decorrentes do exercício, contribui para uma melhora significativa na autoestima, o que leva a um maior grau de satisfação e felicidade ${ }^{42,43}$. Além disso, geralmente a atividade física é uma oportunidade importante para o convívio social, trazendo benefícios fundamentais presentes no estabelecimento de interações afetivas. Sendo assim, a prática de atividade física é vista pela comunidade médica como importante agente preditor ou terapêutico para depressão ${ }^{44,45}$.

A ansiedade também foi associada estatisticamente ao sexo feminino, com exceção do curso de Biomedicina. O mesmo foi detectado em estudos que avaliaram estudantes da área da saúde em cidades brasileiras e fora do Brasil ${ }^{46-48}$. Esses resultados são semelhantes aos encontrados na população geral, que apontam o sexo feminino como aquele em que mais comumente se encontram altos níveis de ansiedade, seja qual for a idade.

Outro aspecto também fortemente associado a maior prevalência de ansiedade foi ter relacionamento insatisfatório com familiares, amigos, colegas de sala e professores $(\mathrm{p}<0,05)$. Andrade et al. ${ }^{32}$, estudando alunos de Medicina, relataram que problemas pessoais ou familiares contribuíam para aumentar o sofrimento mental desses estudantes. Outro trabalho que avaliou estudantes do Rio de Janeiro sugeriu que problemas pessoais e de relacionamento com familiares eram motivos para baixo rendimento acadêmico e aumento da ansiedade e depressão ${ }^{33}$. Maior prevalência de ansiedade nesses alunos com problemas de relacionamento revela um caráter possivelmente sistêmico, evidenciando a necessidade de maior equilíbrio em todas as esferas da vida do estudante. Esse equilíbrio deverá proporcionar melhores condições para a formação adequada desses estudantes, já que problemas nos relacionamentos acabam por gerar dificuldades psicológicas, como insegurança, tristeza, medos, preocupações, dificuldades de atenção e baixa autoestima.

Um dos aspectos mais fortemente associados à ansiedade foi a baixa quantidade de sono, sendo a insônia uma das principais características referidas pelos alunos avaliados. Outro trabalho realizado com estudantes universitários residentes na cidade de Fortaleza apontou que aqueles que tinham médias mais baixas de horas de sono diárias apresentaram índices de ansiedade mais elevados ${ }^{49}$. Não resta dúvida de que a quantidade inadequada de sono pode diminuir o entusiasmo pela execução de atividades cotidianas e pode trazer outros problemas de saúde, podendo ser a ansiedade uma condição presente nessas situações. Nossos achados nessa área refor- 
çam a importância e necessidade de estimular os estudantes a buscar destinar o tempo adequado ao sono e com qualidade, para garantir que esse sono seja reparador.

Outro aspecto que merece destaque foi a baixa prevalência de ansiedade entre os estudantes que referiram fazer atividade física com frequência. Entretanto, esse resultado não se confirmou para o curso de Medicina. Já há evidência de que a realização de atividade física regular influencia a qualidade de vida de estudantes universitários do Nordeste ${ }^{50}$. Contudo, embora a literatura científica aponte os inúmeros benefícios do não sedentarismo para a saúde física e mental, o resultado inverso na Medicina talvez possa ser explicado pela possível angústia gerada pelo acúmulo de atividades e pela falta de tempo. Esse sentimento do estudante acaba gerando a percepção de que o tempo dedicado à realização de atividade física regular pode causar prejuízos acadêmicos, já que a graduação exige tempo integral.

A busca por acompanhamento psicológico deve ser uma prática estimulada entre os estudantes, principalmente aqueles da área da saúde. A ansiedade, por exemplo, foi um dos principais motivos relatados por residentes em Radiologia do Rio de Janeiro para buscar atendimento por um psicólogo ${ }^{33}$. Os trabalhos aqui discutidos apontam a existência de dificuldade de alguns estudantes no enfrentamento de adversidades, o que os levaria a buscar auxílio profissional para superação e melhora do quadro. Essa prática precisa ser fomentada pelas escolas que formam profissionais de saúde para garantir uma formação adequada e, principalmente, humanizada. Há uma vasta literatura disponível que avalia esses aspectos ligados ao estudante de Medicina e o quanto ele sofre de pressão para o ingresso na faculdade, sua formação e o exercício profissional. Entretanto, os dados aqui apresentados reforçam a necessidade de maior reflexão a respeito do olhar que a sociedade tem lançado sobre o profissional de saúde não médico. Não há dúvida quanto às dificuldades por que passa um estudante em sua trajetória para se tornar um médico, mas o resultado em relação aos outros cursos não é desprezível.

\section{CONCLUSÃO}

As prevalências de ansiedade e depressão entre os estudantes da área da saúde foram muito superiores às da população em geral, tendo os estudantes do curso de Fisioterapia apresentado o resultado mais alto. Proporcionar ao estudante da saúde, de qualquer curso, uma formação mais completa e adequada exige repensar desde o processo de ingresso até a oferta de melhores condições de trabalho, passando inclusive por uma formação mais humanizada, na qual o estudante seja atendido em suas necessidades pedagógicas e emocionais. Essa situ- ação aponta a necessidade urgente de maior atenção a esses futuros profissionais, de forma a estarem técnica e emocionalmente mais bem preparados e mais saudáveis para lidar com a saúde humana.

\section{REFERÊNCIAS}

1. WHO. Depression and Other Common Mental Disordes: Global Health Estimates. World Health Organization ed. Geneva2017.

2. OMS. Saúde Mental: Nova Concepção, Nova Esperança. 1 ed. Lisboa: Climepsi; 2002.

3. Mendlowicz MV, Stein MB. Quality of life in individuals with anxiety disorders. American Journal of Psychiatry. 2000;157(5):669-82.

4. Orley J, Kuyken W. Quality of life assessment: international perspectives. Basileia: Springer-Verlag; 1994.

5. Baxter A, Scott K, Vos T, Whiteford H. Global prevalence of anxiety disorders: a systematic review and meta-regression. Psychological medicine. 2013;43(5):897-910.

6. Ferrari AJ, Charlson FJ, Norman RE, Patten SB, Freedman G, Murray CJ, et al. Burden of depressive disorders by country, sex, age, and year: findings from the global burden of disease study 2010. PLoS medicine. 2013;10(11):e1001547.

7. Alves TCdTF. Depressão e ansiedade entre estudantes da área de saúde. Revista de Medicina. 2015;93(3):101-5.

8. Association AP. Manual diagnóstico e Estatístico de Transtornos Mentais- DSM-5. Artmed Editora; 2014.

9. OMS. Classificação dos Transtornos Mentais e de Comportamento da CID-10. Descrições Clínicas e Diretrizes Diagnósticas. Porto Alegre: Artes Médicas; 1993.

10. Sadock BJ, Sadock VA. Manual Conciso de Psiquiatria da Infância e da Adolescência: Kaplan \& Sadock. Porto Alegre: Artmed; 2011.

11. Claudino J, Cordeiro R. Níveis de ansiedade e depressão nos alunos do curso de licenciatura em enfermagem. O caso particular dos alunos da Escola Superior de Saúde de Portalegre. Millenium-Journal of Education, Technologies, and Health. 2016 (32):197-210.

12. STUART A. Ansiedade e Depressão. 1 ed. Lisboa: Climepse; 1993.

13. Hovens JG, Giltay EJ, Wiersma JE, Spinhoven P, Penninx BW, Zitman FG. Impact of childhood life events and trauma on the course of depressive and anxiety disorders. Acta psychiatrica scandinavica. 2012;126(3):198-207.

14. Costello EJ, Egger HL, Angold A. The developmental epidemiology of anxiety disorders: phenomenology, prevalence, and comorbidity. Child and Adolescent Psychiatric Clinics. 2005;14(4):631-48. 
15. da Victoria MS, Bravo A, Felix AK, Neves BG, Rodrigues CB, Ribeiro CCP, et al. Níveis de ansiedade e depressão em graduandos da Universidade do Estado do Rio de Janeiro (UERJ). Encontro: Revista de Psicologia. 2015;16(25):16375.

16. de Rezende CHA, AbrãoI CB, CoelhoI EP, da Silva PassosI LB. Prevalência de sintomas depressivos entre estudantes de medicina da Universidade Federal de Uberlândia. Revista brasileira de educação médica. 2008;32(3):315-23.

17. de Oliva Costa EF, Santana YS, de Abreu Santos ATR, Martins LAN, de Melo EV, de Andrade TM. Sintomas depressivos entre internos de medicina em uma universidade pública brasileira. Revista da Associação Médica Brasileira. 2012:53-9.

18. Vasconcelos TCd, Dias BRT, Andrade LR, Melo GF, Barbosa L, Souza E. Prevalência de Sintomas de Ansiedade e Depressão em Estudantes de Medicina. Revista Brasileira de Educação Médica. 2015;39(1):135-42.

19. de Carvalho EA, Bertolini SMMG, Milani RG, Martins MC. Índice de ansiedade em universitários ingressantes e concluintes de uma instituição de ensino superior/Anxiety scores in university entering and graduating students from a higher education institution. Ciência, Cuidado e Saúde. 2015;14(3):1290-8.

20. Meleiro AMAdS. Suicídio entre médicos e estudantes de medicina. Revista da Associação Médica Brasileira. 1998:44(2):135-40.

21. WONCA MdMdF-, OMS OMdS-. Integração da Saúde Mental nos Cuidados de Saúde Primários: Uma Perspectiva Global. 2008.

22. Cunha JA. Manual da versão em português das Escalas Beck. São Paulo: Casa do Psicólogo; 2001.

23. Beck AT, Beamesderfer A. Assessment of depression: the depression inventory: Karger Publishers; 1974.

24. Beck A, Steer R. Beck anxiety inventory manual. san antonio, tx: The psychological corporation. Harcourt Brace \& Company; 1993.

25. Lantyer AdS, Varanda CC, Souza FGd, Padovani RdC, Viana MdB. Ansiedade e Qualidade de Vida entre Estudantes Universitários Ingressantes: Avaliação e Intervenção. Revista Brasileira de Terapia Comportamental e Cognitiva. 2016;18(2).

26. Galvão A, Pinheiro M, Gomes MJ, Ala S. Ansiedade, stress e depressão relacionados com perturbações do sono-vigília e consumo de álcool. Revista Portuguesa de Enfermagem de Saúde Mental. 2017 (SPE5):8-12.

27. Amaral GFd, Gomide LMdP, Batista MdP, Piccolo PdP, Teles TBG, Oliveira PMd, et al. Sintomas depressivos em acadêmicos de medicina da Universidade Federal de Goiás: um estudo de prevalência. Rev Psiquiatr Rio Gd Sul. 2008;30(2):124-30.

28. Furegato ARF, Nievas AF, Silva EC, Costa Jr ML. Pontos de vista e conhecimentos dos sinais indicativos de depressão entre acadêmicos de enfermagem. Revista da Escola de Enfermagem da USP. 2005;39(4):401-8

29. Carvalho MCP, Junqueira LG, Cerdeira CD, Costa AMDD, Santos GB. Levantamento da situação de saúde mental e uso de ansiolíticos e antidepressivos por acadêmicos do curso de odontologia de uma universidade do sul de Minas Gerais DOI: http:/ /dx. doi. org/10.5892/ruvrd. v15i1. 2772. Revista da Universidade Vale do Rio Verde. 2017;15(1):489-96.

30. Altmann BAR, Campos IFA. As falas das mulheres deprimidas: por uma clínica de tempo, gênero e cultura. 2017.

31. Souza LPS, Barbosa BB, Silva CSdO, Souza AGd, Ferreira TN, Siqueira LdG. Prevalência de transtornos mentais comuns em adultos no contexto da Atenção Primária à Saúde. Revista Portuguesa de Enfermagem de Saúde Mental. 2017 (18):59-66.

32. Andrade JBCd, Sampaio JJC, Farias LMd, Melo LdP, Sousa $\mathrm{DPd}$, Mendonça ALBd, et al. Contexto de formação e sofrimento psíquico de estudantes de medicina. Rev bras educ méd. 2014:231-42.

33. da Silva GCC, KochII HA, de Sousa EG, Gasparetto E, Buys RC. Ansiedade e depressão em residentes em Radiologia e Diagnóstico por Imagem. Revista Brasileira de Educação Médica. 2010;34(2):199-206.

34. de Oliveira GS, Rocha CA, dos Santos BÉF, Sena IS, Favaro L, Guerreiro MC. Prevalência e fatores associados à depressão em estudantes de medicina da Universidade Federal do Amapá. Revista de Medicina e Saúde de Brasília. 2017;5(3)

35. Tabalipa FdO, Souza MFd, Pfützenreuter G, Lima VC, Traebert E, Traebert J. Prevalence of Anxiety and Depression among Medical Students. Revista Brasileira de Educação Médica. 2015;39:388-94.

36. Ramires VRR, Falcke D. Fatores de risco e proteção para vínculos familiares no sul do Brasil. Psicologia: teoria e prática. 2018;20(1):126-40.

37. Teixeira LS, De Oliveira BLG. Uma compreensão sobre a depressão a partir da gestal-terapia. Revista Uningá. 2018;51(2).

38. de Omena Silva A, Neto JLC. Associação entre níveis de atividade física e transtorno mental comum em estudantes universitários / Association between levels of physical activity and common mental disorder in university students. Motricidade. 2014;10(1):49. 
39. Godnho MR, Ferreira AP, dos Santos ASP, da Rocha FSA. Fatores associados à qualidade do sono dos trabalhadores técnico-administrativos em educação de uma universidade pública. Revista de Medicina e Saúde de Brasília. 2018;6(3).

40. Silva PVC, Costa Jr ÁL. Efeitos da atividade física para a saúde de crianças e adolescentes. Psicologia Argumento. 2017;29(64).

41. Costa PHV, da Silva FS, Machado CJ. Nível de atividade física e qualidade de vida dos estudantes de fisioterapia da FCM-MG. Revista Interdisciplinar Ciências Médicas. 2018;2(1):46-53.

42. Bavoso D, Galeote L, Montiel JM, Cecato JF. Motivação e autoestima relacionada à prática de atividade física em adultos e idosos. Revista Brasileira de Psicologia do Esporte. $2018 ; 7(2)$.

43. Almeida LM, Pereira HP, Fernandes HM. Efeitos de diferentes tipos de prática desportiva no bem-estar psicológico de jovens estudantes do ensino profissional. Revista Iberoamericana de Psicología del Ejercicio y el Deporte. 2018;13(1):15-21.

44. Ferreira LWdS. A importância do exercício físico no tratamento de pessoas portadoras de depressão o mal do século. 2017.

45. Pereira DF. Relação entre atividade física e depressão em idosos: uma revisão de literatura. Revista Corpoconsciência. 2017;20(3):22-8.

46. Gama MMA, Moura GS, Araújo RF, Teixeira-Silva F. Ansiedade-traço em estudantes universitários de Aracaju (SE). Revista de Psiquiatria do RS. 2008;30(1):19-24.

47. Loureiro E, McIntyre T, Mota-Cardoso R, Ferreira MA. A relação entre o stress e os estilos de vida nos estudantes de medicina da Faculdade de Medicina do Porto. 2008.

48. Pereira ACM. Análise de depressão e ansiedade nos alunos do ensino superior: comparação com um estudo do curso de radiologia: Escola Superior de Saúde Dr. Lopes Dias; 2009.

49. de Araújo MFM, Lima ACS, Alencar AMPG, de Araújo TM, Fragoaso LVC, Damasceno MMC. Avaliação da qualidade do sono de estudantes universitários de Fortaleza-CE. Texto \& contexto enfermagem. 2013;22(2):352-60.

50. Mendes-Netto RS, da Silva CS, Costa D, Raposo OFF. Nível de atividade física e qualidade de vida de estudantes universitários da área de saúde. Revista de Atenção à Saúde (antiga Rev Bras Ciên Saúde). 2013;10(34).

\section{CONTRIBUIÇÃO DOS AUTORES}

Andrea Mendes Leão participou do desenho do estudo, coleta de dados, redação do artigo e discussão dos resultados. Ileana Pitombeira Gomes participou do desenho do estudo, da coleta de dados e redação do artigo. Marcelo Ferreira participou do desenho do estudo, da análise dos dados e redação do artigo. Luciano Pamplona de Góes Cavalcanti participou do desenho do estudo, da análise dos dados e redação do artigo. Todos os autores aprovaram a versão final do manuscrito.

\section{CONFLITO DE INTERESSES}

Não há.

\section{ENDEREÇO PARA CORRESPONDÊNCIA}

Luciano Pamplona de Góes Cavalcanti

Rua João Cordeiro, 1644 - apto 103

Bairro: Centro - Fortaleza

CEP 60110-300 - Ceará

E-mail: pamplona.luciano@gmail.com 